\title{
Addendum: More extreme precipitation in the world's dry and wet regions
}

\author{
Markus G. Donat, Andrew L. Lowry, Lisa V. Alexander, Paul A. O'Gorman and Nicola Maher \\ Nature Climate Change 6, 508-513 (2016); published online 7 March 2016; corrected after print 1 February 2017.
}

In our Letter we analysed changes in total annual precipitation (PRCPTOT) and extreme precipitation (Rx1day) in the dry and wet regions of the world as defined using precipitation amounts. The estimates of change have been partly biased due to the choice of reference period used for identifying wet and dry regions and normalizing the time series ${ }^{1}$. Here we present our analyses corrected for these biases. While the corrections affect the exact quantification of precipitation changes, the biases are comparable to the uncertainties already discussed in our study, and our conclusions remain unchanged. The corrected results confirm that observations and CMIP5 models show robust increases in precipitation totals and extremes over the past 60 years in the world's dry regions, and in precipitation extremes in the wet regions, while there are only small changes in precipitation totals in the wet regions. Climate projections for the rest of the twenty-first century show that for precipitation over land these observed tendencies are expected to continue into the future, although there are substantial uncertainties about the magnitude of change, in particular in the dry regions.

In our original study, we used the sample mean of the first 30 years of the investigation period (that is, 1951-1980) to normalize the local time series of either precipitation totals or precipitation extremes, and to identify the dry and wet regions. This procedure, however, may artificially increase the spatial mean of the precipitation totals or precipitation extremes outside the climatological reference period because of variability over time in the sample means. Comparable biases related to normalization have previously been discussed for the analysis of temperature time series ${ }^{2,3}$. In addition, although 30 years is the recommended time length to calculate climatologies ${ }^{4}$, it may be too short to derive robust estimates of the spatial distribution of precipitation extremes. As a result, the calculated changes of the dry and wet regions' spatial aggregations may be affected by the 'regression to the mean' phenomenon $^{5,6}$. This means that selecting from the dry part of the spatial distribution may result in a higher probability of wetter conditions outside the reference period, and similarly a higher probability of dry conditions when analysing the wet selection.

To avoid these biases, we use the entire 60 year period 1951-2010 as reference to identify dry and wet regions and to normalize the data. The corrected estimates of change over the 1951-2010 period are shown in Figs 1 and 2 of this Addendum. The increases in the dry regions are somewhat reduced in magnitude, but statistically significant $(p \leq 0.05)$ increases of $1-2 \%$ per decade still occur in both precipitation indices in the dry regions and in the Rxlday index in the wet regions, as reported in our original study. Changes in PRCPTOT in the wet regions remain comparatively small.

For the analysis of model-projected future changes, effects related to the 'regression to the mean' bias were already addressed in our Letter when analysing the future projections by assessing changes in dry and wet regions based on both the past and future period (see section SI5 of the Supplementary Information of the Letter). Avoiding the bias related to normalization of the time series, by avoiding any data out of the reference period, has the disadvantage of using different (and hence incompatible) climatological reference periods for the analyses of past and future changes (Fig. 3). Nevertheless we illustrate here the effect on the analysis of projected future changes when consistently avoiding the discussed biases. For analysing the changes between the future climate period at the end of the twenty-first century (2070-2099) and the past climate period (1951-1980), we combine both the early and the late 30 year periods to calculate a climate average that is used to normalize the time series. Regions are identified as dry only if they are dry according to both the past and future climate periods, and accordingly identified as wet if wet in both periods, as in section SI5 of the Supplementary Information of the Letter.

Also in the future climate projections the rates of change are lower in the dry regions compared to our original study (Fig. 3), with ensemble average increases of $10 \%$ (using all land grid cells) to $18 \%$ (using grid cells with nearly complete observational coverage) for PRCPTOT in the RCP8.5 scenario simulations, and increases of $16 \%$ to $20 \%$ for Rx1day accordingly. When including ocean grid cells the ensemble mean changes in PRCPTOT are close to zero, related to the finding that some dry regions over ocean experience total precipitation decreases in the model simulations. Changes in wet regions are similar to what we reported in our original study. The relationship between dry (and wet) region precipitation changes and the magnitude of global warming in the different model simulations is mostly not statistically significant $(p>0.1)$ for the individual future scenario simulations when using the combined past and future climate reference period to normalize the data. However, there are significant relationships between the changes in global temperature and the regionally aggregated precipitation indices when calculating the linear regression through the data points from both (RCP4.5 and RCP8.5) scenario simulations (Fig. 4).

\section{References}

1. Sippel, S. et al. Have precipitation extremes and annual totals been increasing in the world's dry regions over the last 60 years? Hydrol. Earth Syst. Sci. Discuss. http://dx.doi.org/10.5194/hess-2016-452 (2016).

2. Sippel, S. et al. Quantifying changes in climate variability and extremes: pitfalls and their overcoming. Geophys. Res. Lett. 42, 9990-9998 (2015).

3. Rhines, A. \& Huybers, P. Frequent summer temperature extremes reflect changes in the mean, not the variance. Proc. Natl Acad. Sci. USA 110, E546-E546 (2013).

4. Calculation of Monthly and Annual 30-Year Standard Normals (WMO, 1989).

5. Galton, F. Regression towards mediocrity in hereditary stature. J. Anthropol. Inst. Great Britain Ireland 15, 246-263 (1886).

6. Barnett, A. G., van der Pols, J. C. \& Dobson, A. J. Regression to the mean: what it is and how to deal with it. Int. J. Epidemiol. 34, 215-220 (2004).

\section{Acknowledgements}

We acknowledge S. Sippel, J. Zscheischler, M. Heimann, H. Lange, M. D. Mahecha, G. J. v. Oldenborgh, F. E. L. Otto and M. Reichstein for pointing out the biases related to choice of reference period. 


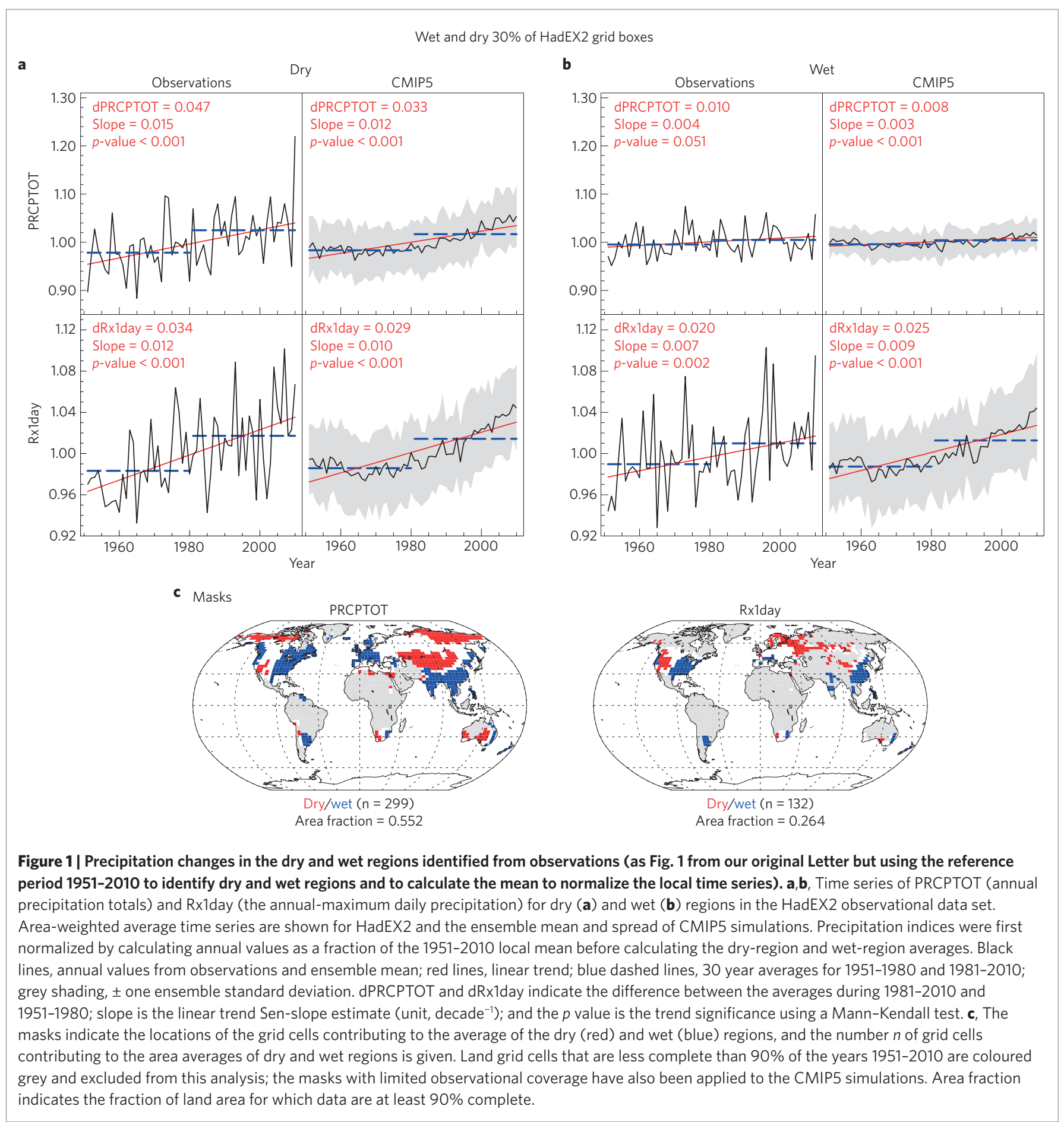




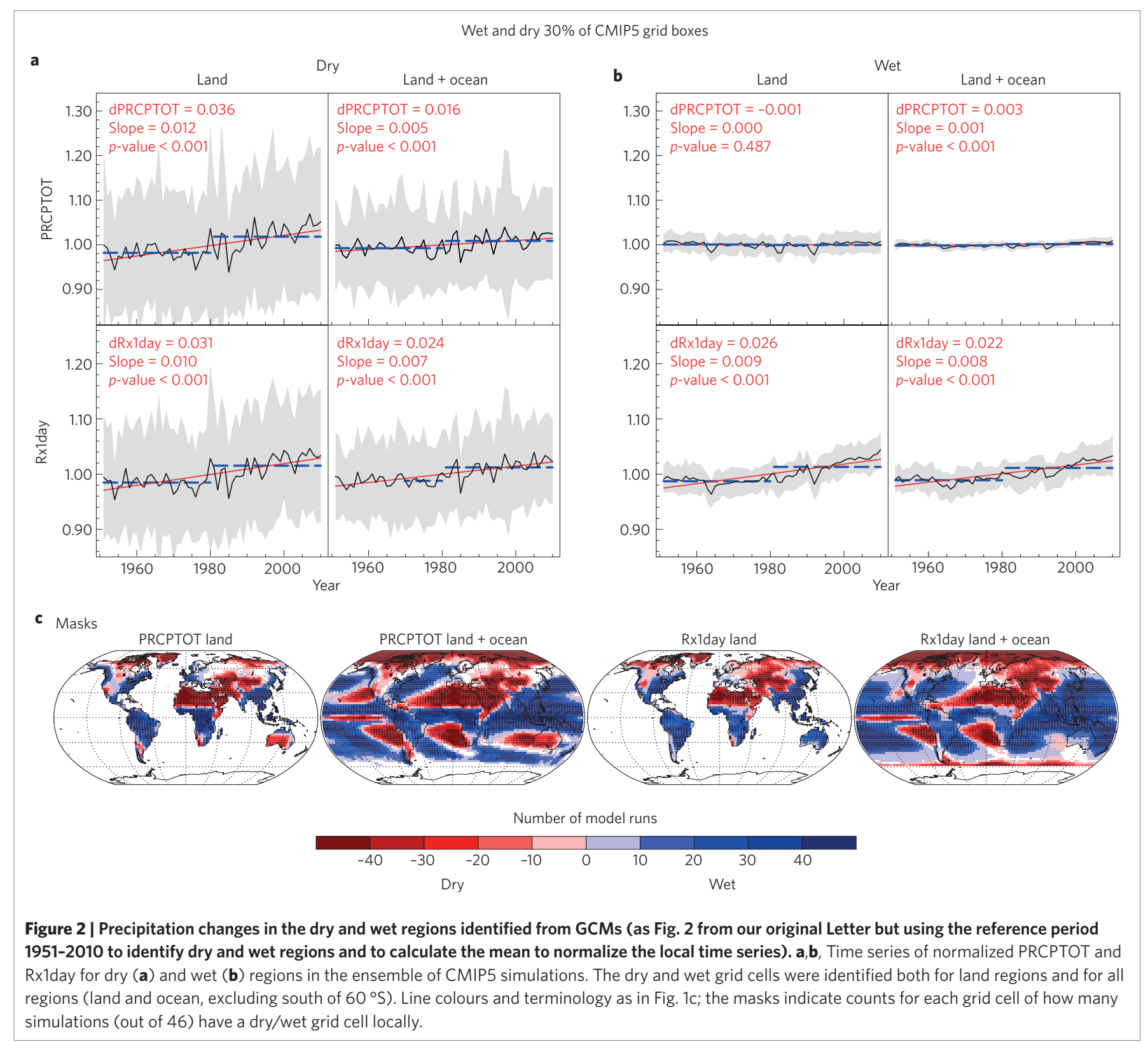




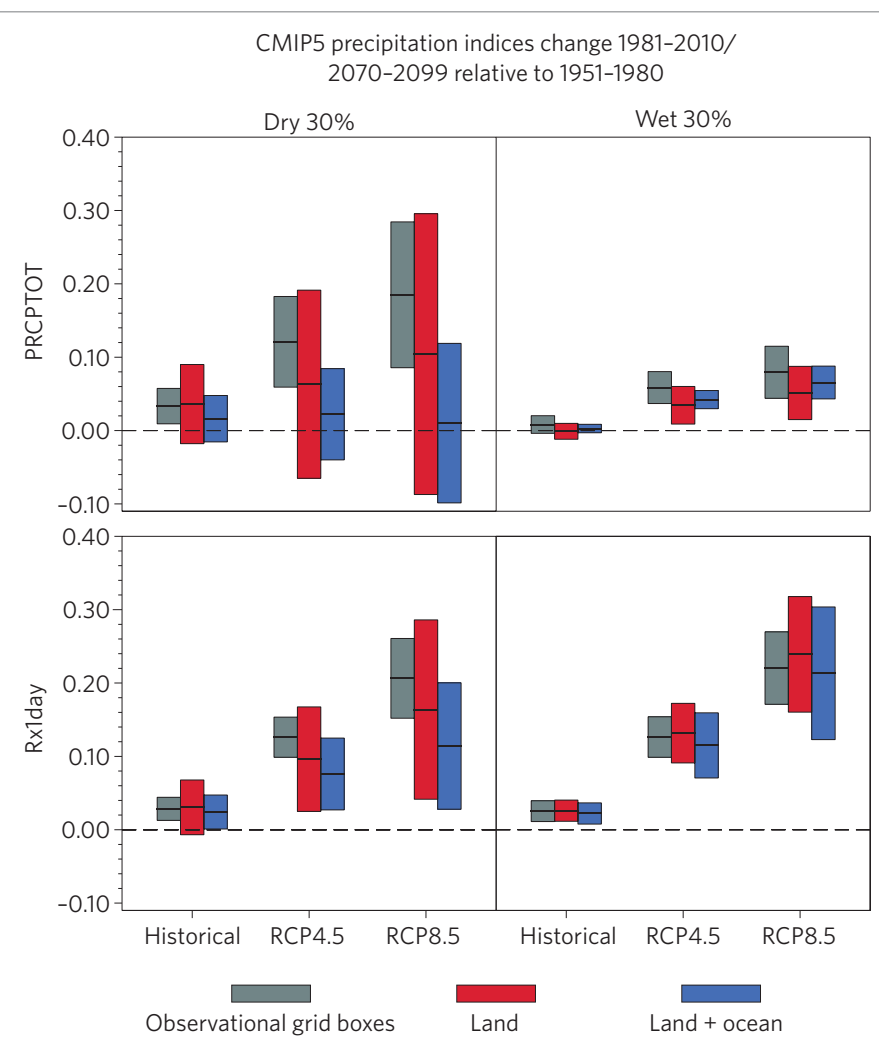

Figure 3 | Simulated precipitation changes in dry and wet regions (as Fig. 3 from our original Letter but using the combined years 1951-1980 plus 2070-2099 as the reference period to normalize the local time series, and the overlap between the dry/wet regions in the past and future period to identify dry and wet regions; for historical changes the reference period 1951-2010 is used). Precipitation index changes averaged over the dry (left column) and wet (right column) regions. Historical changes show the average during 1981-2010, and RCP4.5 and RCP8.5 show the average during 2070-2099, all relative to the 1951-1980 average. Horizontal black lines represent the ensemble-mean changes and the coloured boxes show \pm one ensemble standard deviation. 
PRCPTOT in dry $30 \%$ land

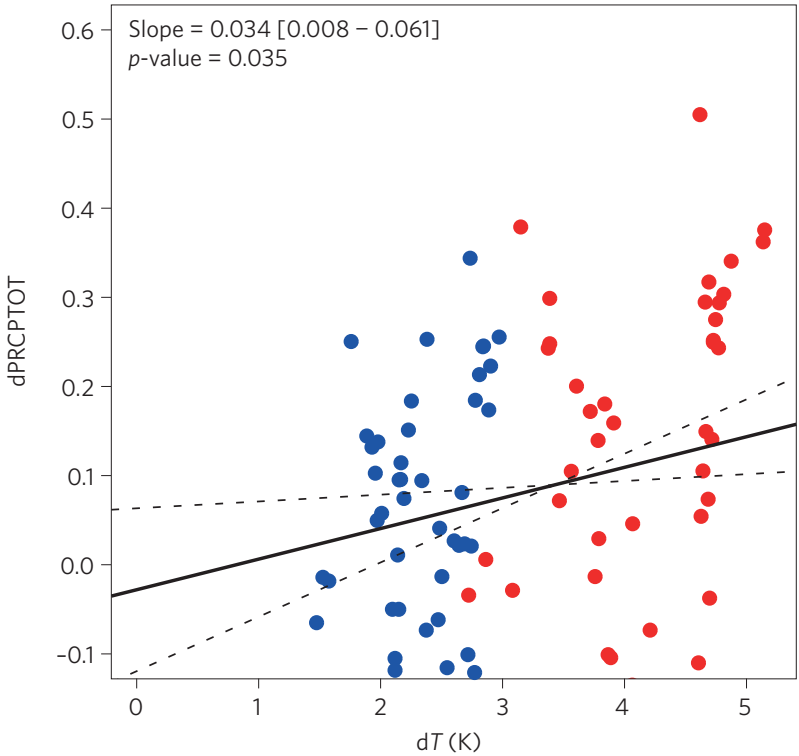

Rx1day in dry $30 \%$ land

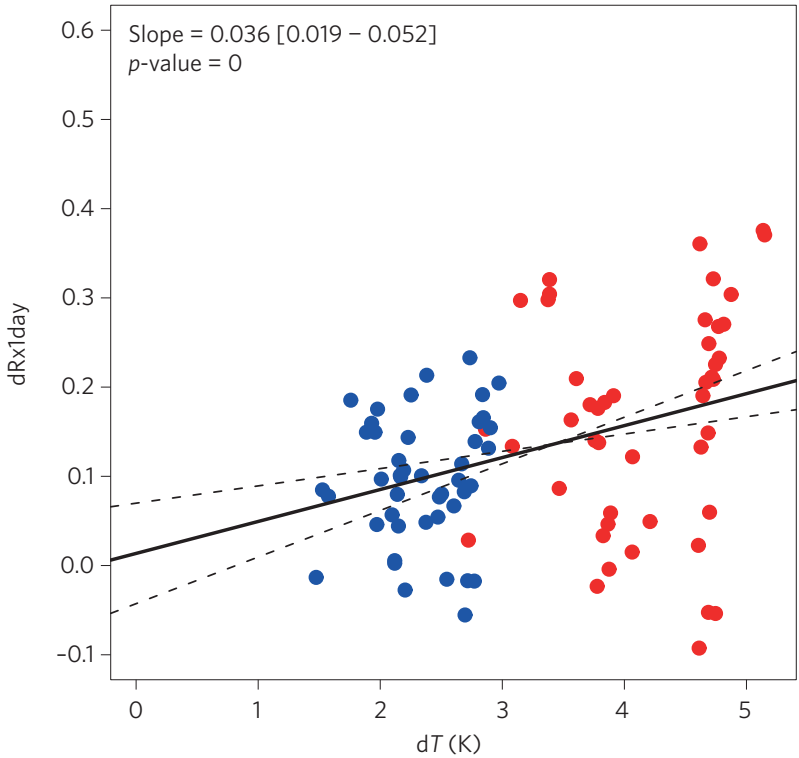

PRCPTOT in wet $30 \%$ land

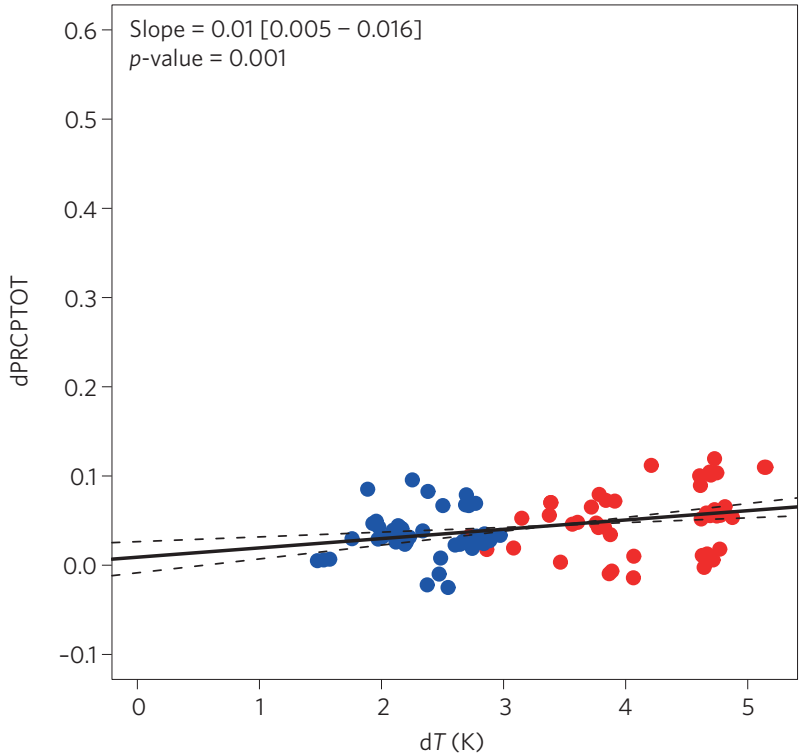

Rx1day in wet $30 \%$ land

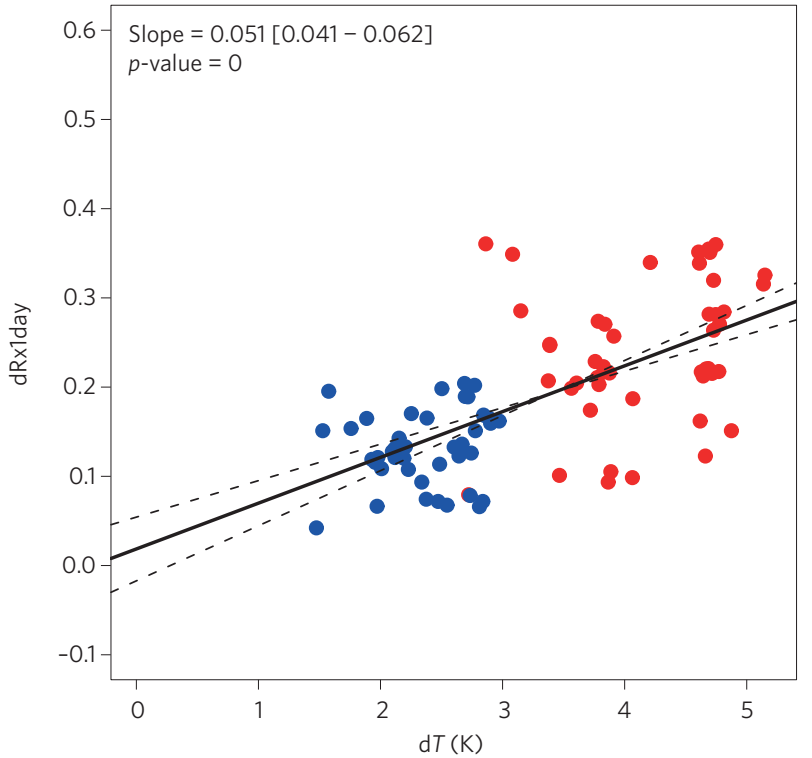

Figure 4 | Precipitation changes as a function of global warming (as Fig. 4 from our original Letter but using the combined years 1951-1980 plus 2070-2099 as the reference period to normalize the local time series, and the overlap between the dry/wet regions in the past and future period to identify dry and wet regions, and showing the regression lines and slopes for all data points). Scatter plots of precipitation index changes over land against global mean temperature change in the different CMIP5 simulations. Precipitation averaged over the dry (left column) and wet (right column) regions. Changes (2070-2099 minus 1951-1980) in RCP4.5 are shown in blue, and RCP8.5 in red. Dots represent the differences in the individual model runs, solid lines represent the linear regression best fit through all data points, and dashed lines represent the 5-95\% uncertainty range of the linear regression slope. 